\title{
Indefinite for Gastric Dysplasia
}

National Cancer Institute

\section{Source}

National Cancer Institute. Indefinite for Gastric Dysplasia. NCI Thesaurus. Code C95758.

A term used to describe an ambiguous morphologic pattern in the gastric mucosa, when there is doubt whether the findings represent a non-neoplastic or neoplastic pathologic process. 